\title{
Incidence of Drug-Drug Interactions among Patients Admitted to the Department of General Medicine in a Tertiary Care Hospital
}

\author{
Namrata Bajracharya, Ann Mary Swaroop, Saraswathy Ganesan Rajalekshmi*, Subeesh K Viswam, Maheswari E \\ Department of Pharmacy Practice, Faculty of Pharmacy, M.S. Ramaiah University of Applied Sciences, Bangalore, Karnataka, INDIA.
}

\begin{abstract}
Background: Drug-Drug Interactions (DDIs) contribute to increased rate of morbidity and mortality increasing the need for intense monitoring of patient safety which can be achieved by detecting and preventing morbidities associated with DDls. Objective and Methodology: The present work was a prospective study carried out for a period of six months, to assess the incidence of DDIs in patients admitted to the Department of General Medicine at a tertiary care hospital. Results: Prescriptions of 411 patients were analysed, out of which $165(40.15 \%)$ prescriptions were identified with potential DDIs whereas clinical manifestations of actual DDIs were observed and reported in $23(5.6 \%)$. A total of 657 DDIs were observed of which $6(0.9 \%), 240(36.5 \%), 374(56.9 \%)$ and $33(5.6 \%)$ were of contraindicated, major, moderate and minor severity respectively. Based on the mechanism $310(47.2 \%)$ of the identified DDIs were pharmacodynamic and $243(36.9 \%)$ were pharmacokinetic interactions. There was a positive correlation between the number of DDIs and risk factors such as
\end{abstract}

length of hospital stay, number of drugs prescribed and co-morbidities. Conclusion: This study concludes that awareness on the most prevalent DDIs can help the practitioners to prescribe drugs with a low risk for DDIs and prevent the concomitant use of dangerous drug combinations.

Key words: Drug-drug interactions, General medicine, Pharmacodynamic, Pharmacokinetic, Severity, Risk factors.

Correspondence

Dr. Saraswathy Ganesan Rajalekshmi, Associate Professor, Department of Pharmacy Practice, Faculty of Pharmacy, M.S. Ramaiah University of Applied Sciences, Bangalore- 560054, Karnataka, INDIA.

Phone: +91 8123230400

Email: saraswathypradish@gmail.com

DOI: 10.5530/jyp.2018.10.98

\section{INTRODUCTION}

Drugs are intended to alleviate disease and improve the quality of life in patients. However, many drugs are reported to cause unwanted reactions ranging from mild rashes to severe adverse reactions with fatal outcomes. Due to the complexity of disease and its comorbidities, multi-drug therapy is the current practice which is found to be alarming as it may result in drug related problems. ${ }^{1}$ Drug-drug interactions represent an important and widely under- recognized source of medication errors and is responsible for $23 \%$ of hospital admissions. ${ }^{2}$ Rational drug utilization may facilitate global reduction in drug induced morbidity and mortality. ${ }^{1}$ Prescriptions with polypharmacy need a thorough evaluation in order to avoid any chance of Drug Related Problems (DRPs) which might result in adverse drug reactions, therapeutic insufficiency and increase the healthcare expenses. The involvement of pharmacist in a health care system may prevent such DRPs. ${ }^{3}$

Administration of two or more drugs may lead to interactions resulting in alteration of therapeutic response or unwanted effects which are not observed with either of the drugs when consumed alone. DDIs may be severe enough to warrant hospital admissions for patients who got it manifested. ${ }^{4-5}$ Ahmad et al, 2015 has reported $66 \%$ of DDIs in the Department of General Medicine at a tertiary care hospital in Karnataka. ${ }^{6}$ Studies have confirmed polypharmacy as one of the major risk factor for the incidence of DDIs. ${ }^{7}$ DDIs contribute $20-30 \%$ incidence of ADRs which may increase the chance of hospital admission or lengthen the hospital stay. ${ }^{8}$ Bhagavathula et al, reported the occurrence of $40 \%$ DDIs in prescriptions with 5 drugs and $80 \%$ with 7 medications or more. ${ }^{9}$
Healthcare organizations must focus on patient safety monitoring for improvised health delivery. The scarcity of national studies on drug interactions and indiscriminate use of drugs, highlight the need for more studies that may contribute for planning and formulation of public health policies in this field. ${ }^{10}$ Therefore, the current study was taken up to improvise the patient safety by monitoring, identifying and preventing DDIs.

\section{MATERIALS AND METHODS}

It is a prospective study conducted to identify the DDIs in patients admitted to the Department of General Medicine at a tertiary care hospital, Bangalore and this study was conducted between January and June 2016. This study was approved Institutional Ethics Committee (IEC) of M.S. Ramaiah Medical College, Bangalore.

\section{Data collection}

The data were retrieved from case sheets, medication charts, laboratory reports and by conducting medication history interviews. The patient profile form was developed which included patient's demographics, history of medications and allergy, diagnosis and clinical laboratory values. DDI form included the details of DDIs with its classifications based on severity, documentation and mechanism.

\section{Data analysis}

DDIs were analysed using Stockley's textbook of drug interactions, Micromedex online database system, Medscape drug interaction checker 
and Drugs.com. Further, DDIs were classified based on the type and severity of interaction as contraindicated, major, moderate and minor along with mechanism of interactions.

\section{Statistical analysis}

Association between factors such as length of hospital stay, number of drugs per prescription, number of comorbidities and DDIs were analysed by chi-square test using SPSS $\mathrm{V}_{20}$.

\section{RESULTS}

In this study, a total of 411patients were enrolled, out of which 243 (59.1\%) were males and 168 (40.9\%) were females (Figure 1). The prescriptions of the enrolled patients were analysed and the maximum number of drugs per prescription of the study population was 22 and minimum number was 2. Among 411 prescriptions, 39 (9.5\%) prescriptions were below 4 medications and $372(90.5 \%)$ were above or equal to 4 medications. The result shows that many prescriptions followed polypharmacy.

Among 411 prescriptions, 165 (40.1\%) were observed with pDDIs and 23 (5.6\%) showed actual DDIs respectively (Figure 2). A total of 657 DDIs were identified in 188 prescriptions. Out of 188 prescriptions with DDIs 123 (65.4\%) prescriptions were in the range of 1-3 DDIs followed by $37(19.7 \%)$ in the range of $4-6$ and 28 (14.9\%) above 6 DDIs (Table 1). 105(25.5\%) DDIs were identified in male whereas $83(20.2 \%)$ were identified in female (Table 2). DDIs were found to be highest among patients aged above 50 years, $106(25.7 \%)$ followed by patients aged between 25-50 years, 62 (15.1\%). The difference in proportion of incidence of DDIs with different age groups was statistically significant $(p<0.05)$ (Table 3$)$. Majority of the study population had co-morbidities along with their primary diagnosis (Figure 3 ).

In this study, the identified DDIs were classified based on the severity, documentation and mechanism (Table 5, 6 and Figure 4). Among the 657 DDIs per prescription, 6 interactions (0.9\%) came under the classification of contraindication, $240(36.5 \%)$ fall under major severity, 374 (56.9\%) were of moderate severity and 37 (5.6\%) were of minor severity. 657 DDIs were analysed for their type or mechanism of interaction. Out of which, 310 were pharmacodynamic DDIs, 243 were

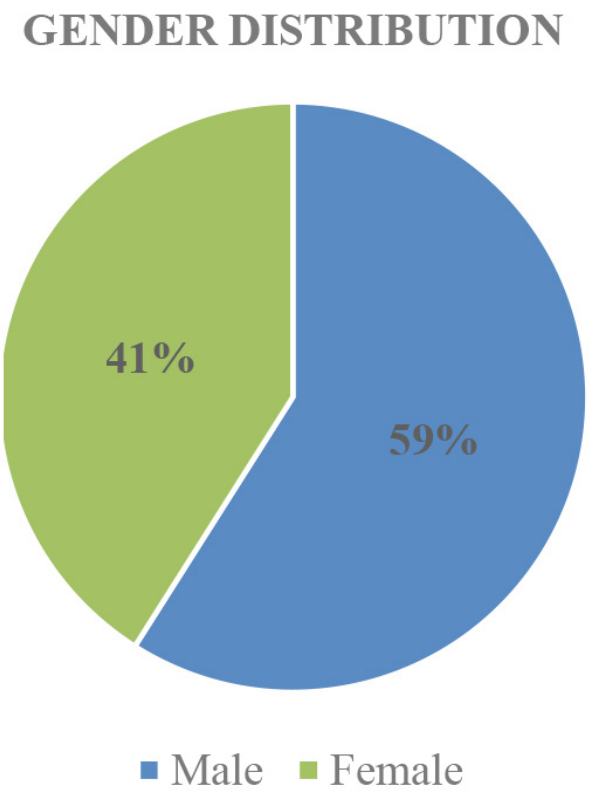

Figure 1: Gender distribution.

\section{PRESENCE OF DDIS}

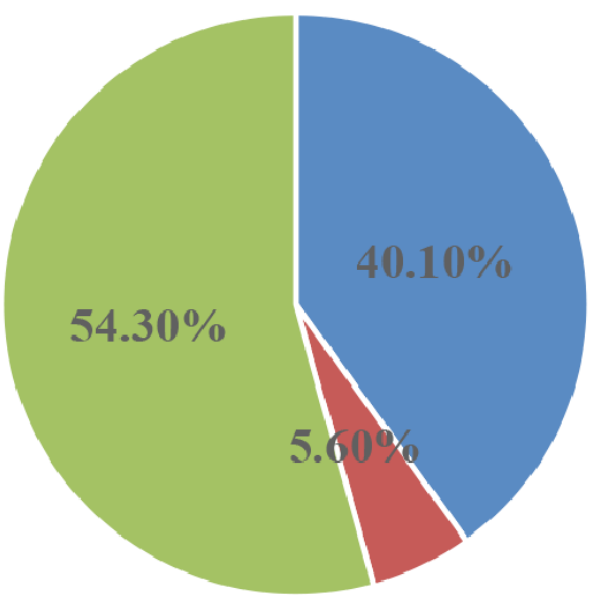

- pDDIs - Actual DDIs - Absent

Figure 2: Presence of DDIs.

\section{COMORBIDITIES}

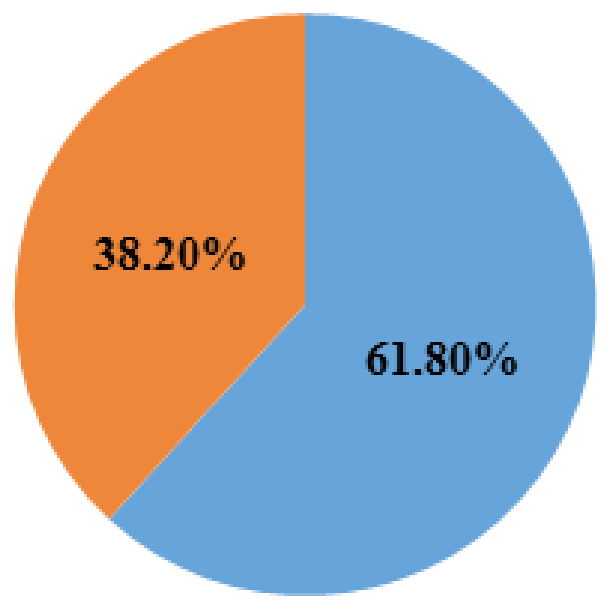

Present Absent

Figure 3: Presence of comorbidities.

pharmacokinetic DDIs. Out of 657 DDIs, 68 (10.4\%) were excellent, 249 (37.9\%) were good and 340 (51.8\%) were fair based on documentation criteria. Mechanism of actual DDIs and most frequently identified pDDIs along with their manifested and anticipated effects are described in Table 7 and 8 respectively. Factors such as length of hospital stay, number of co-morbidities and number of drugs per prescription were analysed and a statistically significant association between occurrence of DDIs and their factors were noted (Table 4). 


\section{MECHANISM OF DDIs}

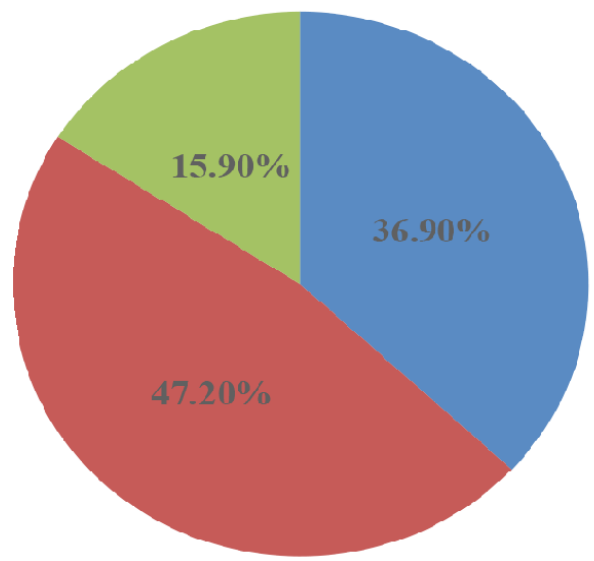

Pharmacokinetic

Pharmacodynamic

Unknown

Figure 4: Mechanism of DDls.

Table 1: Number of DDIs per prescription.

\begin{tabular}{ccc}
\hline Number of DDIs & Number of patients & Percentage (\%) \\
\hline $1-3$ & 123 & $65.4 \%$ \\
$4-6$ & 37 & $19.7 \%$ \\
$>6$ & 28 & $14.9 \%$ \\
Total & 188 & $100 \%$ \\
\hline
\end{tabular}

\section{DISCUSSION}

As the number of medications increase, the complexity of therapy also increases which could lead to DRP and further reduce the clinical outcome. Drug interactions are recognized as the most dangerous DRP. ${ }^{11}$

In this study, majority of the population were males which was similar to a study conducted by Ahmed et al. (2015). ${ }^{6}$ and contrast to the study conducted by Mateti $\mathrm{U}$ et al. ${ }^{12}$

In this study, majority of patients fall under the age group of 25-50 years. The mean age was $45.7 \pm 19$ years. The maximum and minimum age of patients were 88 years and 15 years rspectively. Cruciol-Souza et al. (2006) has reported in their study that the average age of inpatients was $52.7 \pm 18.9$ years ranging from 12 to 98 years. ${ }^{8}$ Our study is online with the studies conducted by Jimmy et al. (2012), Bhagavathula et al. (2014), Nag et al (2011)..$^{9,13,14}$ In this study, there was no appreciable difference in proportion of DDIs among both the genders.

It was found that the maximum number of drugs per prescription was 22 and minimum was 2 . Out of 411 prescriptions, 188 were found with

Table 2: Gender wise distribution of DDIs per prescription.

\begin{tabular}{cccccc}
\hline \multirow{2}{*}{ Range } & \multicolumn{2}{c}{ Male } & \multicolumn{2}{c}{ Female } & \\
\cline { 2 - 5 } & $\begin{array}{c}\text { Number of } \\
\text { patients }\end{array}$ & Percentage & $\begin{array}{c}\text { Number of } \\
\text { patients }\end{array}$ & Percentage & Total \\
\hline $1-3$ & 67 & $35.6 \%$ & 56 & $29.8 \%$ & 123 \\
$4-6$ & 21 & $11.2 \%$ & 16 & $8.5 \%$ & 37 \\
$>6$ & 17 & $9.1 \%$ & 11 & $5.9 \%$ & 28 \\
Total & 105 & $55.9 \%$ & 83 & $44.1 \%$ & 188 \\
\hline
\end{tabular}

The chi square statistic is 0.3758 . The p-value is 0.828694 . The result is not significant at $p<0.05$.

Table 3: Age wise categorization of DDIs per prescription.

\begin{tabular}{|c|c|c|c|c|c|c|c|}
\hline \multirow{2}{*}{$\begin{array}{l}\text { Age group } \\
\text { (years) }\end{array}$} & \multicolumn{2}{|c|}{$1-3$} & \multicolumn{2}{|c|}{$4-6$} & \multicolumn{2}{|l|}{$>6$} & \multirow[b]{2}{*}{ Total } \\
\hline & $\begin{array}{c}\text { Number of } \\
\text { patients }\end{array}$ & Percent & $\begin{array}{c}\text { Number of } \\
\text { patients }\end{array}$ & Percent & Number of patients & Percent & \\
\hline$<25$ & 19 & $10.1 \%$ & 1 & $0.5 \%$ & 0 & $0 \%$ & 20 \\
\hline $25-50$ & 48 & $25.5 \%$ & 10 & $5.3 \%$ & 3 & $1.6 \%$ & 61 \\
\hline$>50$ & 56 & $29.8 \%$ & 26 & $13.8 \%$ & 25 & $13.3 \%$ & 107 \\
\hline Total & 123 & $65.4 \%$ & 37 & $19.6 \%$ & 28 & $14.9 \%$ & 188 \\
\hline
\end{tabular}

The chi square statistic is 23.01 . The $\mathrm{p}$-value is 0.000126 . The result is significant $(p<0.05)$.

Table 4: Predictors associated with the occurrence of DDI.

\begin{tabular}{|c|c|c|c|c|c|c|c|c|c|c|c|c|c|c|}
\hline DDIs & \multicolumn{4}{|c|}{ Length of Hospital Stay in Days } & \multicolumn{5}{|c|}{ Number of Co-morbidities } & \multicolumn{5}{|c|}{ Number of drugs per prescription } \\
\hline 0 & 134 & 83 & 6 & 223 & 93 & 11 & 20 & 13 & 137 & 30 & 42 & 12 & 2 & 86 \\
\hline $4-6$ & 11 & 22 & 24 & 57 & 29 & 13 & 26 & 28 & 96 & 1 & 68 & 42 & 20 & 131 \\
\hline$>6$ & 11 & 34 & 42 & 87 & 1 & 11 & 32 & 44 & 88 & 1 & 31 & 19 & 64 & 115 \\
\hline \multicolumn{5}{|c|}{ Predictors } & \multicolumn{5}{|c|}{ Chi- square statistic } & & \multicolumn{4}{|c|}{$P$ value } \\
\hline \multicolumn{5}{|c|}{ Length of hospital stay } & \multicolumn{5}{|c|}{126.75} & & \multicolumn{4}{|c|}{$<0.001$} \\
\hline \multicolumn{5}{|c|}{ Number of medicines } & \multicolumn{5}{|c|}{116.69} & & \multicolumn{4}{|c|}{$<0.001$} \\
\hline \multicolumn{5}{|c|}{ Concurrent illness } & \multicolumn{5}{|c|}{186.65} & & \multicolumn{4}{|c|}{$<0.001$} \\
\hline
\end{tabular}


Table 5: Severity of DDIs.

\begin{tabular}{cccc}
\hline \multirow{2}{*}{ Severity } & \multicolumn{2}{c}{ Number of DDls } & Percentage (\%) \\
\cline { 2 - 3 } & pDDIs & Actual DDIs & \\
\hline Contraindicated & 6 & 0 & $0.9 \%$ \\
Major & 219 & 21 & $36.5 \%$ \\
Moderate & 366 & 8 & $56.9 \%$ \\
Minor & 36 & 1 & $5.6 \%$ \\
Total & 627 & 30 & $100 \%$ \\
\hline
\end{tabular}

Table 6: Documentation of DDIs.

\begin{tabular}{cccc}
\hline \multirow{2}{*}{ Documentation } & \multicolumn{2}{c}{ Number of DDls } & $\%$ of DDls \\
\cline { 2 - 3 } & pDDls & Actual DDIs & \\
\hline Excellent & 68 & 0 & $10.4 \%$ \\
Good & 243 & 6 & $37.9 \%$ \\
Fair & 326 & 24 & $51.8 \%$ \\
Total & 627 & 30 & $100 \%$ \\
\hline
\end{tabular}

Table 7: Mechanism of identified actual DDIs and the effect produced.

\begin{tabular}{cccccc}
\hline Interactions & Frequency & Severity & Documentation & Mechanism & Effect produced \\
\hline Quetiapine + Carbamazepine & 2 & Major & Fair & Pharmacokinetic & $\begin{array}{c}\text { Increased drowsiness and reduced Quetiapine } \\
\text { concentration }\end{array}$ \\
Pyrazinamide + Rifampin & 1 & Major & Good & Unknown (additive) & Hepatic injury (ALP increased) \\
Oxcarbazepine + Tolvaptan & 1 & Major & Fair & Pharmacokinetic & Reduced tolvaptan concentration and hyponatremia \\
& & & & & (123.5mEq) \\
Aspirin+ Calcium carbonate & 2 & Moderate & Fair & Pharmacokinetic & Decreased salicylate effect \\
Aspirin + Hydrocortisone & 1 & Minor & Good & Pharmacodynamic & Gastric ulceration \\
Aspirin + Clopidogrel & 6 & Major & Fair & Pharmacodynamic & GI bleeding \\
Cefotaxime + Warfarin & 1 & Major & Good & Unknown & Increased INR (3.5) \\
Metalazone + Torsemide & 2 & Major & Good & Pharmacodynamic & Hyponatremia (122, 120.9mEq/L) \\
Digoxin + Aspirin & 1 & Major & Good & Pharmacokinetic & Hyperkalemia (6.1mEq/L) \\
Heparin + Enoxaparin & 3 & Major & Fair & Pharmacodynamic & Bleeding manifestation \\
Furosemide + Metoprolol & 2 & Moderate & Fair & Pharmacodynamic & Hypokalemia (2.5, 2.7mEq/L) \\
Furosemide + Albuterol & 2 & Moderate & Fair & Pharmacodynamic & Hypoglycaemia (GRBS- 65, 91 mg/dl) \\
Metformin + Insulin aspart & 2 & Moderate & Fair & Pharmacodynamic & Seizures \\
Levofloxacin + Tramadol & 1 & Major & Fair & Pharmacodynamic & Prolongation of QT interval \\
Ondansetron + Levofloxacin & 3 & Major & Fair & Pharmacodynamic &
\end{tabular}

Table 8: Mechanism of most frequently identified pDDIs and their anticipated effects.

\begin{tabular}{|c|c|c|c|}
\hline \multicolumn{2}{|c|}{ Interactions } & \multirow{2}{*}{$\frac{\text { Frequency }}{4}$} & \multirow{2}{*}{$\begin{array}{c}\text { Anticipated effects } \\
\text { Increased risk of QT prolongation }\end{array}$} \\
\hline Contraindicated & Fluconazole+Ondansetron & & \\
\hline & Clarithromycin+Ivabradine & 1 & Increased ivabradine exposure and risk of QT prolongation \\
\hline & Clarithromycin+Fluconazole & 1 & Increased Clarithromycin exposure and risk of cardiotoxicity \\
\hline \multirow[t]{6}{*}{ Major } & Clopidogrel+Aspirin & 37 & Increased risk of bleeding \\
\hline & Enoxaparin+Aspirin & 19 & Increased risk of bleeding \\
\hline & Azithromycin+Ondansetron & 15 & Increased risk of QT prolongation \\
\hline & Clopidogrel+Enoxaparin & 14 & Increased risk of bleeding \\
\hline & Amlodipine+Clopidogrel & 11 & Decreased antiplatelet effect and increased risk of thrombotic effect \\
\hline & Metronidazole+Ondansetron & 10 & Increased risk of QT interval prolongation and arrhythmia \\
\hline \multirow[t]{7}{*}{ Moderate } & Atorvastatin + Clopidogrel & 26 & Decreased formation of clopidogrel active metabolite \\
\hline & Furosemide + Aspirin & 23 & Decreased diuretic and antihypertensive efficacy \\
\hline & Iron+Pantoprazole & 22 & Reduced iron bioavailability \\
\hline & Aspirin+Insulin & 15 & Increase the risk of hypoglycemia \\
\hline & Atorvastatin+Azithromycin & 13 & Increased risk of rhabdomyolysis \\
\hline & Aspirin + Spironolactone & 11 & Decreased diuretic effectiveness hyperkalemia or nephrotoxicity \\
\hline & Aspirin+Ramipril & 10 & Reduced ramipril effectiveness \\
\hline \multirow[t]{3}{*}{ Minor } & Folic acid+Nitrofurantoin & 5 & Decreased folic acid serum level \\
\hline & Aspirin+Ranitidine & 4 & Decreased salicylate blood levels and antiplatelet effect of aspirin \\
\hline & Aspirin + Phenytoin & 3 & Decreased phenytoin concentrations \\
\hline
\end{tabular}


Bajracharya, et al.: Drug Interactions among General Medicine Inpatients

the DDIs. A total of 657 DDIs were found with an average of 3.49 DDIs per patients. Our work revealed that the overall prevalence of DDIs were $46 \%$ and the prevalence rate of DDIs were reported to be $49.7 \%, 56.2 \%$, $63 \%$ and $78 \%$ were reported by Cruciol-Souza et al. (2006), Vonbach et al. (2008), Umretiya et al. (2015) and Bhagavathula et al. (2014) respectively. ${ }^{8-9,15-16}$ The study showed that the incidence rate of DDIs were more in males than females, this may be because maximum of the study population were males. These findings were similar to the studies carried out by Umretiya et al. (2015) and Nag et al. (2011). ${ }^{13,16}$ whereas in contrast to the studies by Lubinga et al. (2011), Jimmy et al.(2012) and Moura et al. (2009) which reported high incidence rate of DDIs in females. ${ }^{7,17,14}$

Age distribution revealed the incidence rate of DDIs to be highest among patients aged above 50 years which could be attributed to the fact that the number of co morbidities are more in older patients which leads to polypharmacy in this population and it has further increased the chances of developing DDIs. The studies conducted by Bista et al. (2009), Merlo et al. (2001), Jimmy et al. (2012) showed that the incidence rate of DDIs was $36 \%$ in the age range of $46-60$ years and $30.95 \%$ in the age above 60 years. ${ }^{14,18,19}$ Whereas the study conducted by Ismail et al. (2011), Lubinga et al. (2011), Nag et al. (2011) reported incidence rate of DDIs more in the age range of $30-40$ years. ${ }^{7,13,20}$ Here, the ranges for the DDIs were categorized as: 1-3, 4-6 and >6. Among 188 prescriptions with DDIs, 1-3 DDIs were found in maximum prescriptions. There was no statistically significant difference between male and female with respect to incidence of DDIs.

In 411 prescriptions, 188 (45.7\%) prescriptions had at least one interacting drug combination. Among the 657 DDIs, majority were classified as moderate which is comparable with the results of Ahmad et al. (2015) where the major, moderate and minor pDDIs were 44 (31.65\%), 75 (53.95\%) and $20(14.38 \%)$ respectively. ${ }^{6}$

Most of the DDIs were fair in documentation which is similar to the work of Jimmy et al. (2012) who reported the documentation of DDIs to be fair 165 (50\%), good $134(40.61 \%)$ and excellent 31 (9.39\%). ${ }^{14}$

657 DDIs were analyzed for their type or mechanism of interaction. Out of which, maximum were pharmacodynamic mechanism. Lubinga et al. (2011) revealed that the majority of DDIs were postulated to occur through pharmacodynamic mechanism followed by pharmacokinetic. In our study the most commonly interacting drug combination was found to be aspirin and clopidogrel 43 (6.5\%), which was expected to increase the risk of bleeding manifestations.

As the length of hospital stay increased, the number of medicines also escalated, which further increased the chance of occurrence of DDIs. In our study there was a positive association between the length of hospital stay, number of comorbidities, number of drugs per prescription and the number of DDIs $(p<0.001)$. Similar associations were observed by Sharma et al. (2014) among cardiac patients in a teaching hospital in Nepal. ${ }^{21}$

Out of 188 prescriptions, 23 of them showed 30 clinical manifestations and upon reporting of those DDIs by Clinical pharmacist few drug combinations were withdrawn from the regimen and appropriate management was done. Among those manifestations, the serious reactions were QT prolongation and bradycardia due to Ondansetron and Levofloxacin combination in a 31 year old female patient admitted in MICU and manifestation of seizures due to Tramadol and Levofloxacin combination in a 19 year old female. In both the cases the interacting drug levofloxacin was withdrawn and substituted with suitable alternatives, which resulted in improvement of the above conditions.

\section{CONCLUSION}

Our study concludes that the incidence rate of DDIs is high at the study site. Majority of the patients received polypharmacy. The identified predictors responsible for DDIs were polypharmacy, age, duration of hospital stay and the number of comorbidities. Hence, it is important to develop a systemic approach to minimize the possible DDIs. Clinical relevance of certain DDIs might be because of their pharmacological actions. The clinical pharmacist is of prime importance to provide information for a better decision on therapy, improve quality of treatment and reduce risks in the patients.

This study tries to put forward the common DDIs which we came across in tertiary care hospitals and this may be a forewarning to health care team about reactions that may occur due to an interaction, as well as provide a support material for physicians to choose an alternate therapy, dose adjustments and patient monitoring. Most often the consequences of DDIs can be managed by withdrawal of potential drugs, specific symptomatic treatments, using alternative drug or dose adjustments. Awareness on the most prevalent DDIs can help the practitioners prescribe drugs with a low risk for DDIs and thereby prevent the concomitant use of dangerous medication combinations.

\section{LIMITATION}

Long-time follow up of the patients was not possible because of which delayed onset DDIs could not be assessed.

\section{ACKNOWLEDGEMENT}

We sincerely thank our Dean, Dr. V. Madhavan for giving us an opportunity and for his constant support during our project work.

\section{CONFLICT OF INTEREST}

The authors declare no conflict of interest.

\section{ABBREVIATIONS}

DDIs: Drug-Drug Interactions; DRPs: Drug related problems; IEC: Institutional Ethics Committee.

\section{REFERENCES}

1. Swamy R, Ramani J, Bushipaka R, Radadiya M, Sowmya B, Patel D. Prevalence of Polypharmacy and Drug to Drug Interactions in a Tertiary Care Teaching Hospital at Karnataka, India. Int. Res. J. Pharm. 2014; 5(10):778-2.

2. Malkesh M, Alpa G. Potential Drug-Drug Interactions among Prescribed Drugs in Paediatric Outpatients Department of a Tertiary Care Teaching Hospital. J Young Pharm. 2017;9(3):371-5.

3. Rajakannan T, Mallayasamy S, Guddattu V, Kamath A, Vilakkthala R, Rao PG, et al. Cost of adverse drug reactions in a South Indian tertiary care teaching hospital. J Clin Pharmacol. 2012; 52(4):559-65.

4. Lal HM, Lal U. Drug Interactions - Mechanisms and Clinical Implications, Medicine Update; 18(ch-89). 2008;18:674-90.

5. Baxter K, Stockley IH. Stockley's drug interactions: A source book of interactions, their mechanisms, clinical importance and management, London: Pharmaceutical Press. 2008.

6. Ahmad A, Khan MU, Haque I, Ivan R, Dasari R, Revanker M, et al. Evaluation of Potential Drug - Drug Interactions in General Medicine Ward of Teaching Hospital in Southern India. J Clin Diagn Res. 2015;9(2):10-3.

7. Lubinga S, Uwiduhaye E. Potential Drug-Drug Interactions on In-patient Medication Prescriptions at Mbarara Regional Referral Hospital (MRRH) in Western Uganda: Prevalence, Clinical Importance and Associated Factors. Afr Health Sci. $2011 ;(3): 499-507$.

8. Cruciol-Souza JM, Thomson JC. Prevalence of potential drug-drug interactions and its associated factors in a Brazilian teaching hospital. J Pharm Pharmaceut Sci. 2006:9(3):427-33. 
9. Bhagavathula AS, Berhanie A, Tigistu H, Abraham Y, Getachew Y, Khan TM, et al. Prevalence of Potential Drug-Drug Interactions among Internal Medicine Ward in University of Gondar Teaching Hospital, Ethiopia. Asian Pac J Trop Biomed. 2014;4(1):204-8.

10. Noblat B, Noblat B, Toledo D, Santos M, Oliveira D, Tanajura GM, et al. Prevalence of Hospital Admission due to Adverse Drug Reaction in Salvador, Bahia. Rev Assoc Med Bras. 2010:57(1):42-5.

11. Hamilton RA, Briceland $L L$, Andritz $M H$. Frequency of Hospitalization after Exposure to Known Drug-Drug Interactions in a Medicaid Population. Pharmacotherapy: J Human Pharmacol Drug Ther. 1998;18(5):1112-20.

12. Mateti UV, Rajakannan T, Nekkanti H, Rajesh V, Mallaysamy SR, Ramachandran P. Drug-drug Interactions in Hospitalized Cardiac Patients. J Young Pharm. 2011;3(4):329-33.

13. Nag KA, Umesh M, Churi S. Assessment of Drug-Drug Interactions in Hospitalised Patients in India. Asian J Pharm Clin Res. 2011:4(1):62-5.

14. Jimmy OD, Shobha Rani RH, Indira R, Ramjan S. Study of Drug-drug Interactions in the Medication Charts in Medicine Wards at a Tertiary Care Hospital, Bangalore. Indian J Pharm Practice. 2012:5(4):61-4.

15. Vonbach P, Dubied A, Krahenbuhl S, Beer JH. Prevalence of Drug-Drug Interactions at Hospital Entry and during Hospital Stay of Patients in Internal Medicine at Switzerland. Eur J Intern Med. 2008:19(6):413-20.

16. Umretiya T, Patil NR, Manjunath G, Kumar C. Assessment of Potential DrugDrug Interactions in the Department of Medicine at Basaweshwara Teaching and General Hospital Gulbarga. J Pharm Sci. 2015:5(3):90-6.

17. Moura C, Acurcio F, Belo N. Drug-Drug Interactions associated with Length of Stay and Cost of Hospitalization in Brazil. J Pharm Pharmaceut Sci. 2009: 12(3):266-72.

18. Bista D, Saha A, Mishra P, Palaian S, Shankar PR. Pattern of Potential Drug-Drug Interactions in the Intensive Care Unit of a Teaching Hospital in Nepal: A Pilot Study, J ClinDiagn Res. 2009:8(3):1713-6.

19. Merlo J, Liedholm H, Lindblad U, Bjorck-Linne A, Fal J, Lindberg G, et al. Prescriptions with Potential Drug Interactions Dispensed at Swedish Pharmacies in January 1999: Cross Sectional Study. Br Med J. 2001:323(7310):427-8.

20. Ismail M, labal Z, Khattak MB, Javaid A, Khan TM. Prevalence, Types and Predictors of Potential Drug-Drug Interactions in Pulmonology Ward of a Tertiary Care Hospital in Pakistan. Afr J Pharm Pharmacol. 2011:5(10):1303-9.

21. Sharma S, Chhetri HP, Alam K. A study of potential drug-drug interactions among hospitalized cardiac patients in a teaching hospital in Western Nepal. Indian J Pharmacol. 2014:46(2):152.

Article History: Submission Date : 14-02-2018; Revised Date : 07-04-2018; Acceptance Date : 15-04-2018.

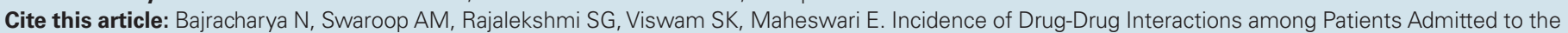
Department of General Medicine in a Tertiary Care Hospital. J Young Pharm. 2018;10(4):450-5. 